\title{
Análise de estabilidade de sistemas incertos através do critério de routh
}

\author{
Stability analysis of uncertain systems \\ through routh's criterion
}

\author{
Márcio Roberto Covacic ${ }^{1}$; Marcelo Carvalho Minhoto Teixeira ${ }^{2}$; \\ Edvaldo Assunção ${ }^{2}$
}

\section{Resumo}

O Critério de Estabilidade de Routh é uma ferramenta simples e útil para a análise da estabilidade de sistemas de controle. Neste trabalho, é proposto um método para o estudo da estabilidade de sistemas incertos $d x(t) / d t=\left(A_{o}+\alpha \Delta A\right) x(t)$, utilizando o Critério de Estabilidade de Routh na análise dos autovalores de $A_{o}+\alpha \Delta A$. O método determina a faixa de valores de $\alpha$ que garante a estabilidade assintótica do sistema. Uma aplicação análoga para sistemas com realimentação negativa do tipo $d x(t) / d t=(A-B K C)$ $x(t), \operatorname{com} K=k I$, também é proposta.

Palavras-chave: Estabilidade. Sistemas Incertos. Critério de Routh. Realimentação da Saída. Polinômio Característico.

\begin{abstract}
Routh's Stability Criterion is a simple and useful tool for stability analysis of control systems. In this work, it is proposed a method In this work, it is proposed a method for stability study of uncertain systems $d x(t) / d t=\left(A_{o}+\alpha \Delta A\right) x(t)$, using Routh's Stability Criterion in the analysis of the eigenvalues of $A_{o}+\alpha \Delta A$. The method determines the range of values of $a$ that guarantees the asymptotic stability of the system. An analog application for negative output feedback systems given by $d x(t) / d t=(A-B K C) x(t)$, where $K=k I$, is also presented.

Key words: Stability. Uncertain Systems. Routh's Criterion. Output Feedback. Characteristic Polynomial.
\end{abstract}

1 Doutor em Engenharia Elétrica, Faculdade de Engenharia de Ilha Solteira, Universidade Estadual Paulista - FEIS-UNESP; Docente do Departamento de Engenharia Elétrica da Universidade Estadual de Londrina - DeEL-uel; e-mail: marciocovacic@uel.br

2 Docentes do Departamento de Engenharia Elétrica da Faculdade de Engenharia de Ilha Solteira, Universidade Estadual Paulista - DEE-FEIS-UNESP; e-mail: marcelo@feis.unesp.br; edvaldo@feis.unesp.br 


\section{Introdução}

$\mathrm{Na}$ teoria de controle clássico, entre as diversas especificações de um sistema de controle, a estabilidade é a mais importante. Em vista disso, existem alguns métodos que podem ser utilizados no estudo da estabilidade de sistemas realimentados com controladores proporcionais, tais como o método do lugar das raízes, o critério de estabilidade de Nyquist e o Critério de Estabilidade de Routh.

$\mathrm{Na}$ maioria dos cursos de controle ministrados para a graduação, o Critério de Estabilidade de Routh é o primeiro método a ser ensinado. Este fato pode ser observado em quase todos os livros sobre teoria de controle clássico (KUO, 1995; CHEN, 1993; D’AZZO; HOUPIS, 1995; DORF; BISHOP, 1998; FRANKLIN; POWELL; EMAMI-NAEINI, 1994; OGATA, 2003; ROWLAND, 1986), nos quais o Critério de Estabilidade de Routh é introduzido imediatamente após a definição de estabilidade.

Um sistema de controle com uma função de transferência $G(s)=n(s) / d(s)$ é assintoticamente estável, se e somente se todos os seus pólos possuírem parte real negativa. Por meio do Critério de Estabilidade de Routh, esta condição pode ser verificada sem a necessidade da determinação das raízes de $d(s)$.

Uma aplicação básica do Critério de Estabilidade de Routh é determinação do conjunto de valores de um ganho escalar $k \in \mathbb{R}$ que estabiliza um sistema com uma entrada e uma saída (em inglês, Single Input Single Output, SISO) com realimentação da saída. Esta aplicação é abordada em (TEIXEIRA; ASSUNÇÃO; COVACIC, 2007), que também estende os resultados para controladores Proporcionais-Integrais-Derivativos (PID) e propõe um programa em Matlab para determinar as regiões de estabilidade para os controladores abordados.

Várias aplicações do Critério de Estabilidade de Routh estão disponíveis na literatura. Em (BLONDEL; LUNDVALL, 1995), o critério é utilizado para verificar se um sistema pode ser estabilizado com um controlador estável. Em (PEÑA, 2003), são apresentados testes para verificar as condições de Routh-Hurwitz e a positividade total de uma matriz, transformando-a em uma matriz triangular superior. Em (HWANG; YANG, 2001), os autores utilizam as condições de Routh-Hurwitz para verificar a propriedade Hurwitz (se todas as raízes possuem parte real negativa) de um segmento de polinômios $(1-\lambda) p_{0}(s)+\lambda p_{l}(s)$, sendo que $0 \leq \lambda$ $\leq 1$. Em (BOSE, 1989; YANG; HWANG, 2002), o método é estendido para combinações convexas de polinômios complexos. Em (BARMISH, 1984; BIAŁAS; GARLOFF, 1985; GUIVER; BOSE, 1983), é apresentado um método para a análise da estabilidade de polinômios, cujos coeficientes sofrem perturbações. Em (BANDYOPADHYAY; ISMAIL; GOREZ, 1994; BANDYOPADHYAY; UPADHYE; ISMAIL, 1997; HWANG; YANG, 1999), o método é estendido para a obtenção de modelos de intervalo de ordem reduzida para intervalos de sistemas lineares, sendo que cada coeficiente de $n(s)$ e $d(s)$ pertence a um intervalo. Em (WEI; YEDAVALLI, 1987), os coeficientes do polinômio caraterístico dependem de um conjunto de parâmetros, com cada parâmetro pertencente a um intervalo, tal que os coeficientes dependem um do outro. Os autores propõem um método para eliminar a dependência entre os parâmetros.

Em Teixeira, Assunção e Covacic (2007), o Critério de Estabilidade de Routh é utilizado para determinar a faixa de valores de um parâmetro $k \in \mathbb{R}$, tal que todas as raízes, $s \in \mathbb{C}$, de um polinômio característico $d(s, k)$, dependente de $k$, possuam parte real negativa. O polinômio característico $d(s, k)$ é dado por:

$$
d(s, k)=d_{n}(k) s^{n}+\ldots+d_{1}(k) s+d_{0}(k),
$$

cujos coeficientes $d_{i}(k), i=1,2, \ldots, n$, são polinômios em $k$, como descrito em (2): 


$$
d_{i}(k)=d_{i b_{-} i} k^{b i}+\ldots+d_{i l} k+d_{i 0^{\circ}}
$$

para $i=0, \ldots, n$, sendo b_i o grau de $d_{i}(k)$.

Neste artigo, o Critério de Estabilidade de Routh será utilizado para a análise da estabilidade de sistemas incertos $d x(t) / d t=\left(A_{o}+\alpha \Delta A\right) x(t)$, sendo $A_{o}$ e $\Delta A$ matrizes constantes e conhecidas e $\alpha \in \mathbb{R}$ um parâmetro constante incerto. Por meio do estudo dos autovalores de $A_{o}+\alpha \Delta A$, é determinada a faixa de valores de $\alpha$ que garante a estabilidade assintótica do sistema. De forma análoga, será analisada a estabilidade de sistemas com realimentação negativa do tipo $d x(t) / d t=(A-B K C) x(t)$, com $K=k I$, sendo $I$ uma matriz identidade, cuja ordem corresponde ao número de entradas, que é supostamente igual ao número de saídas do sistema.

Este artigo é organizado como segue. A Seção 2 mostra o Critério de Estabilidade de Routh e apresenta o método proposto em (TEIXEIRA; ASSUNÇÃO; COVACIC, 2007) para a análise da estabilidade de sistemas de controle. A Seção 3 descreve a utilização do Critério de Estabilidade de Routh para a análise da estabilidade de sistemas incertos $d x(t) / d t=\left(A_{o}+\alpha \Delta A\right) x(t)$ e uma aplicação análoga para sistemas realimentados $d x(t) / d t=(A$ $B K C) x(t)$, com $K=k I$. A Seção 4 mostra alguns exemplos de aplicação. A Seção 5 é uma conclusão deste trabalho.

\section{Estabilidade de polinômios com dependência polinomial}

Considere um sistema linear invariante no tempo $G(s, k)=n(s, k) / d(s, k)$, cujo polinômio característico $d(s, k)$ é dado por (1), sendo $d_{n}(k), \ldots d_{l}(k)$ e $d_{0}(k)$ polinômios em $k$, descritos em (2), para $i=0, \ldots, n$, sendo $b_{i}$ o grau do coeficiente $d_{i}(k)$.

Considere que o grau de $d(s, k)$ é constante, isto é, $d_{n}(k) \neq 0$. O sistema é estável, se e somente se todas as raízes, com relação à variável $s \in C$, do polinômio característico $d(s, k)$ tiverem parte real negativa. O Critério de Estabilidade de Routh (OGATA, 2003) é uma ferramenta útil para a análise da estabilidade.

Por meio do Critério de Estabilidade de Routh, podem-se obter os valores de $k \in \mathbb{R}$ tais que todas as raízes do polinômio característico $d(s, k)$ possuam parte real negativa, caso existam. Considere a Tabela 1.

Tabela 1. Tabela de Routh.

\begin{tabular}{cc|ccc} 
linha $n$ & $s^{n}$ & $a_{n 1}(k)=\frac{p_{n 1}(k)}{q_{n}(k)}$ & $a_{n 2}(k)=\frac{p_{n 2}(k)}{q_{n}(k)}$ & $a_{n 3}(k)=\frac{p_{n 3}(k)}{q_{n}(k)}$ \\
linha $(n-1)$ & $s^{n-1}$ & $a_{(n-1) 1}(k)=\frac{p_{(n-1) 1}(k)}{q_{(n-1)}(k)}$ & $a_{(n-1) 2}(k)=\frac{p_{(n-1) 2}(k)}{q_{(n-1)}(k)}$ & $a_{(n-1) 3}(k)=\frac{p_{(n-1) 3}(k)}{q_{(n-1)}(k)}$ \\
$\vdots$ & $\vdots$ & $\vdots$ & $\vdots$ \\
linha 2 & $s^{2}$ & $a_{21}(k)=\frac{p_{21}(k)}{q_{2}(k)}$ & $a_{22}(k)=\frac{p_{22}(k)}{q_{2}(k)}$ \\
linha 1 & $s^{1}$ & $a_{11}(k)=\frac{p_{11}(k)}{q_{1}(k)}$ & \\
linha 0 & $s^{0}$ & $a_{01}(k)=\frac{p_{01}(k)}{q_{0}(k)}$ &
\end{tabular}


Os termos da linha $n$ são dados por $a_{n I}(k)$, $a_{n 2}(k), \ldots$, sendo:

$$
\begin{gathered}
\mathrm{p}_{\mathrm{n} 1}(\mathrm{k})=\mathrm{d}_{\mathrm{n}}(\mathrm{k}), \mathrm{p}_{\mathrm{n} 2}(\mathrm{k})=\mathrm{d}_{(\mathrm{n}-2)}(\mathrm{k}), \\
\mathrm{p}_{\mathrm{n} 3}(\mathrm{k})=\mathrm{d}_{(\mathrm{n}-4)}(\mathrm{k}), \\
q_{n}(\mathrm{k})=1 .
\end{gathered}
$$

Os termos da linha (n-1) são dados por $a_{(n-1) 1}(k)$, $a_{(n-1) 2}(k), \ldots$, sendo:

$$
\begin{gathered}
p_{(n-1) l}(k)=d_{(n-1)}(k), \quad p_{(n-1) 2}(k)=d_{(n-3)}(k), \\
q_{(n-l)}(k)=1 .
\end{gathered}
$$

Para determinar estas duas primeiras linhas, seguindo o procedimento acima, considera-se que $d_{i}(k)=0$, para $i=-1,-2,-3, \ldots$

Os termos da linha $j, j=n-2, \ldots, 1,0$, são dados por:

$$
\begin{aligned}
a_{j i}(k) & =\frac{a_{(j+1) 1}(k) a_{(j+2)(i+1)}(k)-a_{(j+2) 1}(k) a_{(j+1)(i+1)}(k)}{a_{(j+1) 1}(k)}, \\
a_{j i}(k) & =\frac{\frac{p_{(j+1) 1}(k)}{q_{(j+1)}(k)} \frac{p_{(j+2)(i+1)}(k)}{q_{(j+2)}(k)}-\frac{p_{(j+2) 1}(k)}{q_{(j+2)}(k)} \frac{p_{(j+1)(i+1)}(k)}{q_{(j+1)}(k)}}{\frac{p_{(j+1) 1}(k)}{q_{(j+1)}(k)}} .
\end{aligned}
$$

Então, $a_{j i}(k)=p_{j i}(k) / q_{j}(k)$, sendo:

$$
\begin{gathered}
p_{j i}(k)=p_{(j+1) l}(k) p_{(j+2)(i+1)}(k)-p_{(j+2) l}(k) p_{(j+1)(i+l)}(k), \\
q_{j}(k)=q_{(j+2)}(k) p_{(j+1) I}(k), \\
j=n-2, n-1, \ldots, 1,0, \quad i=1,2, \ldots
\end{gathered}
$$

Os elementos não nulos $p_{j i}(k)$ e $q_{j}(k), j=n, n$ $1, \ldots, 1,0, i=1,2, \ldots$, são polinômios em $k$. De acordo com o Critério de Estabilidade de Routh, o número de raízes de $d(s, k)$ com parte real positiva é igual ao número de mudanças de sinal na primeira coluna da tabela de Routh. Então, para um dado valor de $k=k_{o}$, a condição necessária e suficiente para que todas as raízes de $d\left(s, k_{o}\right)$ tenham parte real negativa é que todos os elementos $a_{j l}\left(k_{o}\right), j=n, n-1, \ldots, 1,0$, da primeira coluna da tabela de Routh apresentem o mesmo sinal.

Em Teixeira, Assunção e Covacic (2007), foi proposto um método para a determinação da faixa de valores de $k \in \mathbb{R}$ tais que todas as raízes de $d(s, k)$ apresentem parte real negativa, a partir das raízes reais dos termos $p_{j}, j=n, \ldots, 1,0$, da primeira coluna da tabela de Routh. Este método é descrito a seguir.

Sejam $z_{j l}, z_{j 2}, z_{j 3} \ldots, z_{j l j}$ as raízes reais de $p_{j l}(k)$ e $q_{j}(k)$, tais que $z_{j 1}<z_{j 2}<z_{j 3}<\ldots<z_{j l j}$, sendo as raízes repetidas consideradas somente uma vez, $l_{-} j$ o número de raízes reais distintas de $p_{j l}(k)$ e $q_{j}(k)$ e $j=n, n-1, \ldots, 1,0$. Note que o sinal de $a_{j l}(k)$ pode mudar, em função de $k \in \mathbb{R}$, somente nas raízes reais de $p_{j l}(k)$ e $q_{j}(k)$, para $k=z_{j i}, i=1,2, \ldots, l j$. Nos intervalos entre essas raízes, definidos por $z_{j i}<k<z_{j(i+1)}$, para $i=1,2, \ldots, l_{j-1}, k<z_{j l}$ e $k>z_{j l j}$, o sinal de $a_{j l}(k)$ é constante.

Analisando todos os elementos na primeira coluna da tabela de Routh, a variação de sinal ocorre somente nas raízes dos termos $p_{j 1}(k)$ e $q_{j}(k)$, $j=n, n-1, \ldots, 1,0$. Note, em (3), (4) e (5), que $q_{n}(k)$ e $q_{(n-1)}(k)$ não possuem raízes e que as raízes de $q_{j}(k)$, $j=0,1,2, \ldots, n-2$, são também raízes de $p_{(n-1) 1}(k), p_{(n-}$ ${ }_{2) I}(k), \ldots p_{I I}(k)$. Portanto, basta considerar somente as raízes de $p_{n I}(k), p_{(n-1) l}(k), \ldots, p_{0 I}(k)$.

Sejam $z_{l}, z_{2}, z_{3}, \ldots, z_{l}$ as raízes reais de todos os termos $p_{j l}(k), j=n, n-1, \ldots, 1,0$, tais que $z_{1}<z_{2}<z_{3}<\ldots$ $<z_{l}$, sendo as raízes repetidas consideradas somente uma vez, e sendo $l$ o número de raízes reais distintas de todos os termos $p_{j l}(k)$ na primeira coluna da tabela de Routh. Nos intervalos entre essas raízes, 
os sinais de todos os elementos da primeira coluna são constantes. Então, para a analisar a estabilidade do sistema, basta considerar um ponto de cada intervalo, de acordo com Figura 1.

Seja $k=m_{i}$ um ponto qualquer do intervalo $I_{i}$. Se todos os termos $a_{j 1}\left(m_{i}\right), j=n, n-1, \ldots, 1,0$, na primeira coluna da tabela de Routh, apresentam o mesmo sinal, então todas as raízes de $d\left(s, m_{i}\right)$ apresentam parte real negativa e, assim, o sistema é estável para qualquer valor de $k$ no intervalo $I_{i}$. Se o sistema é instável para todos os pontos $\mathrm{m}_{\mathrm{i}}, \mathrm{i}=1,2, \ldots, 1$, então o sistema é instável para qualquer valor de $k$ e o problema de estabilização de malha fechada não tem solução.

Observação 1. (TEIXEIRA; ASSUNÇÃO; COVACIC, 2007) Uma possivel escolha dos pontos $\mathrm{m}_{\mathrm{i}}$ é dada abaixo:

$$
\begin{gathered}
m_{0}= \begin{cases}2 z_{1}, & \text { se } z_{1}<0, \\
-1, & \text { se } z_{1} \geq 0,\end{cases} \\
m_{i}=\frac{z_{i}+z_{i+1}}{2}, i=1,2, \ldots, l-1, \\
m_{l}= \begin{cases}2 z_{l}, & \text { se } z_{l}>0, \\
1, & \text { se } z_{l} \leq 0 .\end{cases}
\end{gathered}
$$

\section{Análise da estabilidade de sistemas incertos}

O método apresentado na seção anterior é útil na análise da estabilidade de sistemas incertos como o sistema definido por:

$$
d x(t) / d t=A x(t)=\left(A_{0}+\alpha \Delta A\right) x(t)
$$

sendo $A_{0}$ e $\Delta A \in \mathbb{R}{ }^{n \times n}$ matrizes conhecidas e $\alpha \in \mathbb{R}$ um parâmetro desconhecido.

A condição necessária e suficiente para a estabilidade assintótica do sistema (6) é que todos os autovalores de $A=A_{0}+\alpha \Delta A$ possuam parte real negativa. Os autovalores de $A$ são os valores de $s \in C$ tais que:

$$
d(s, \alpha)=\operatorname{det}(s I-A)=\operatorname{det}\left(s I-A_{o}-\alpha \Delta A\right)=0 .
$$

O polinômio característico $d(s, \alpha)$ pode ser determinado pelos Lemas 1 e 2, propostos em (TEIXEIRA; MARCHESI; ASSUNÇÃO, 2001):

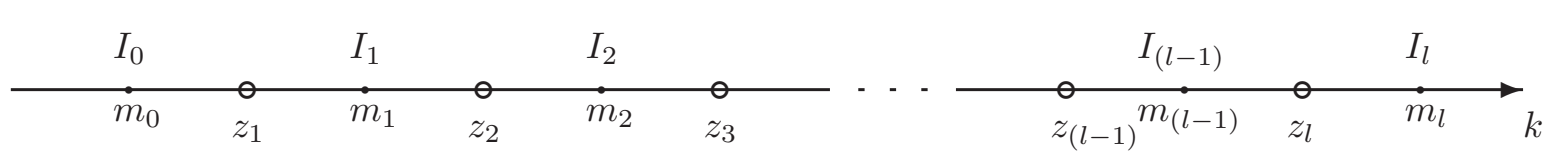

Figura1. Intervalos entre as raízes reais dos termos da primeira coluna. 
Lema 1. (TEIXEIRA; MARCHESI; ASSUNÇÃO, 2001) Considere a matriz $M(\delta) \in \mathbb{R}^{\mathrm{m} \times \mathrm{m}}$, dada por $M(\delta)=\delta_{i} M_{1}+\ldots+\delta_{n} M_{n}$. As matrizes $M_{i}, i=1, \ldots, n$, podem ser decompostas da seguinte forma:

$$
M_{i}=\left[\begin{array}{c}
a_{i 1} \\
a_{i 2} \\
\vdots \\
a_{i m}
\end{array}\right], i=1, \ldots, n
$$

Assim, o determinante de $M(\delta)$, indicado por $|M(\delta)|$, pode ser determinado por:

$$
|M(\delta)|=\sum_{s_{1}=1}^{n} \cdots \sum_{s_{m}=1}^{n} \delta_{s_{1}} \cdots \delta_{s_{m}}\left|\begin{array}{c}
a_{s_{1} 1} \\
a_{s_{2} 2} \\
\vdots \\
a_{s_{m} m}
\end{array}\right| .
$$

Lema 2. (TEIXEIRA; MARCHESI; ASSUNÇÃO, 2001) Considere a seguinte matriz $M \in \mathbb{R}^{\mathrm{n} \times \mathrm{n}}$ :

$$
\begin{gathered}
M=\left[\begin{array}{cccc}
m_{11} & m_{12} & \cdots & m_{1 n} \\
m_{21} & m_{22} & \cdots & m_{2 n} \\
\vdots & \vdots & \ddots & \vdots \\
m_{n 1} & m_{n 2} & \cdots & m_{n n}
\end{array}\right] \\
=m_{11}\left[\begin{array}{cccc}
1 & 0 & \cdots & 0 \\
0 & 0 & \cdots & 0 \\
\vdots & \vdots & \ddots & \vdots \\
0 & 0 & \cdots & 0
\end{array}\right]+\ldots+m_{n n}\left[\begin{array}{cccc}
0 & 0 & \cdots & 0 \\
0 & 0 & \cdots & 0 \\
\vdots & \vdots & \ddots & \vdots \\
0 & 0 & \cdots & 1
\end{array}\right]
\end{gathered}
$$$$
\text { e defina: }
$$$$
\begin{aligned}
h_{s_{i}} & =\left[\begin{array}{lll}
h_{s_{i}, 1} \cdots h_{s_{i}, i-1} & h_{s_{i}, i} & h_{s_{i}, i+1} \cdots h_{s_{i}, n}
\end{array}\right] \\
& =\left[\begin{array}{lll}
0 \cdots 0 & 1 & 0 \cdots 0
\end{array}\right], i=1, \ldots, n .
\end{aligned}
$$

Então, o determinante de $M$ pode ser descrito por:

$$
|M|=\sum_{s_{1}=1}^{n} \sum_{\substack{s_{2}-1 \\
s_{2} \neq s_{1}}}^{n} \cdots \sum_{\substack{s_{n}-1 \\
s_{n} \neq s_{1}, \ldots, s_{n-1}}}^{n} m_{1, s_{1}} \cdots m_{n, s_{n}}\left|\begin{array}{c}
h_{s_{1}} \\
\vdots \\
h_{s_{n}}
\end{array}\right| .
$$

Obtido o polinômio característico $d(s, \alpha)$, através dos Lemas 1 e 2, a faixa de valores de $\alpha$ para os quais o sistema (6) é estável pode ser determinada através do método proposto na seção anterior.

Observação 2. Na expressão de d( $(\mathrm{\alpha}, \mathrm{\alpha})$, o coeficiente de $s^{n}$ é constante e igual a 1. Logo, o grau de $d(s, \alpha)$ é igual a $n$, para qualquer valor de $\alpha \in \mathbb{R}$.

Observação 3. Do Lema 1 e de (7), note que sI$A_{o}-\alpha \Delta A=\delta_{1} M_{1}+\delta_{2} M_{2}+\delta_{3} M_{3}$, sendo que $\delta_{1}=s, \delta_{2}=1$, $\delta_{3}=\alpha, M_{1}=I, M_{2}=-A_{o}$ e $M_{3}=-\Delta A$. Assim, $\operatorname{det}\left(\operatorname{sI}-A_{o}-\right.$ $\alpha \Delta A$ ) pode ser obtido utilizando-se (8).

Observação 4. Note que as matrizes $\left[\begin{array}{llll}h_{s_{-}}{ }^{T} & \ldots & h_{s_{-} n}\end{array}\right]$ ${ }^{T}$ em (9) são independentes de s e $\alpha$ e suas linhas correspondem às linhas da matriz identidade, ordenadas de maneiras diferentes. Logo, os determinantes dessas matrizes são iguais a 1 ou -1. Assim, o polinômio característico $d(s, \alpha)$ pode ser obtido facilmente e sem a utilização de cálculos simbólicos, que requerem um grande esforço computacional.

Análise da estabilidade de sistemas com realimentação negativa

O método proposto nesta seção também pode ser utilizado para a análise da estabilidade de sistemas com realimentação negativa da saída, como o sistema indicado na Figura 2, com $K=k I$.

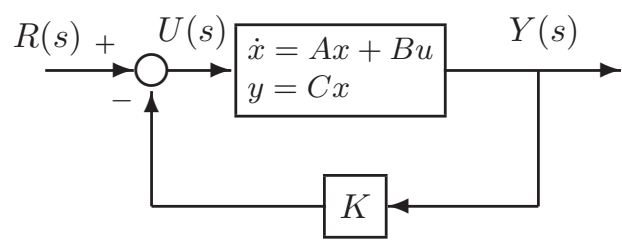

Figura 2. Sistema com realimentação negativa. 
Considere a planta definida por (10):

$$
\begin{gathered}
d x(t) / d t=A x(t)+B u(t), \\
y(t)=C x(t),
\end{gathered}
$$

sendo $A \in \mathbb{R}^{n \times n}, B \in \mathbb{R}^{n \times m}, C \in \mathbb{R}^{m \times n}$, e a lei de controle $u(t)$ dada por:

$$
u(t)=-K y(t)+r(t)
$$

com $K=k I$. Então, o sistema realimentado $\{A-B K C, B, C\}$ da Figura 2 é definido por (12):

$$
\begin{gathered}
d x(t) / d t=(A-B K C) x(t)+B r(t)= \\
=(A-k B C) x(t)+B r(t) .
\end{gathered}
$$

O sistema (12) é estável se e somente se todos os autovalores da matriz característica $(A-k B C)$ tiverem parte real negativa. Os autovalores de $(A-k B C)$ são os valores de $s \in \mathbb{C}$ tais que:

$$
d(s, k)=\operatorname{det}(s I-(A-k B C))=\operatorname{det}(s I-A+k B C) .
$$

A faixa de estabilidade do sistema pode ser determinada de forma análoga à análise da estabilidade de sistemas incertos proposta nesta seção, considerando, neste caso, $A_{o}=A, \Delta A=-B C$ e $\alpha=k$.

\section{Exemplos}

\section{Exemplo 1}

Considere o sistema incerto definido por (6), com:

$$
\begin{gathered}
A_{0}=\left[\begin{array}{ccc}
0 & 1 & 0 \\
0 & 0 & 1 \\
-1 & -2 & -1
\end{array}\right], \\
\Delta A=\left[\begin{array}{ccc}
0 & 0 & 0 \\
0 & 0 & 0 \\
1 & -1 & 1
\end{array}\right]
\end{gathered}
$$

A matriz do sistema $A=A_{0}+\alpha \Delta A$ é dada por:

$$
A=\left[\begin{array}{ccc}
0 & 1 & 0 \\
0 & 0 & 1 \\
-1+\alpha & -2-\alpha & -1+\alpha
\end{array}\right]
$$

e o polinômio característico, calculado por meio dos Lemas 1 e 2 e da Observação 3, é:

$$
d(s, \alpha)=s^{3}+(1-\alpha) s^{2}+(2+\alpha) s+(1-\alpha) .
$$

Os valores de $\alpha$ que tornam estável o sistema incerto (6), com as matrizes $A_{o}$ e $\Delta A$ dadas em (13), são determinados através do Critério de Estabilidade de Routh, com a tabela abaixo:

$$
\begin{array}{l|ll}
s^{3} & a_{31}(\alpha)=\frac{p_{31}(\alpha)}{q_{3}(\alpha)} & a_{32}(\alpha)=\frac{p_{32}(\alpha)}{q_{3}(\alpha)} \\
s^{2} & a_{21}(\alpha)=\frac{p_{21}(\alpha)}{q_{2}(\alpha)} & a_{22}(\alpha)=\frac{p_{22}(\alpha)}{q_{2}(\alpha)} \\
s^{1} & a_{11}(\alpha)=\frac{p_{11}(\alpha)}{q_{1}(\alpha)} & \\
s^{0} & a_{01}(\alpha)=\frac{p_{01}(\alpha)}{q_{0}(\alpha)} &
\end{array}
$$

cujos termos $p_{j i}(\alpha)$ e $q_{j}(\alpha), j=3,2,1,0, i=1,2$, são dados na Tabela 2. 
Tabela 2. Termos da Tabela de Routh para o Exemplo 1.

\begin{tabular}{|c|c|c|c|}
\hline$j$ & $p_{j 1}(\alpha)$ & $p_{j 2}(\alpha)$ & $q_{j}(\alpha)$ \\
\hline 3 & 1 & $2+\alpha$ & 1 \\
\hline 2 & $1-\alpha$ & $1-\alpha$ & 1 \\
\hline 1 & $1-\alpha^{2}$ & 0 & $1-\alpha$ \\
\hline 0 & $1-\alpha-\alpha^{2}+\alpha^{3}$ & 0 & $1-\alpha^{2}$ \\
\hline
\end{tabular}

As raízes reais dos termos $p_{j 1}(\alpha)$, na primeira coluna da tabela de Routh, são dadas na Tabela 3 .

Tabela 3. Raízes reais dos termos $\mathrm{p}_{\mathrm{j} 1}(\alpha)$.

\begin{tabular}{|c|c|}
\hline$j$ & Raízes reais de $p_{j 1}(\alpha)$ \\
\hline 3 & - \\
\hline 2 & $z_{21}=1$ \\
\hline 1 & $z_{11}=-1, z_{12}=1$ \\
\hline 0 & $z_{01}=-1, z_{02}=1, z_{03}=1$ \\
\hline
\end{tabular}

Assim, as raízes reais dos termos $p_{j l}(\alpha)$, na primeira coluna da tabela de Routh, em ordem crescente, sem repetição, são:

$$
z_{1}=-1, z_{2}=1
$$

As raízes acima dividem o conjunto dos números reais em três intervalos. Para a análise da estabilidade do sistema, basta analisar um ponto de cada intervalo, conforme a Figura 1. De acordo com a Observação 1, os pontos escolhidos são:

$$
m_{0}=-2, m_{1}=0, m_{2}=2
$$

Para os pontos acima, os valores numéricos dos termos da primeira coluna da tabela de Routh são dados na Tabela 4.
Tabela 4. Valores numéricos dos termos $a_{\mathrm{j} 1}(k)$ para os pontos escolhidos dos intervalos.

\begin{tabular}{|c|c|c|c|}
\hline$i$ & 0 & 1 & 2 \\
\hline Intervalo & $k<-1$ & $-1<k<1$ & $k>1$ \\
\hline$m_{i}$ & -2 & 0 & 2 \\
\hline$a_{31}\left(m_{i}\right)$ & 1 & 1 & 1 \\
\hline$a_{21}\left(m_{i}\right)$ & 3 & 1 & -1 \\
\hline$a_{11}\left(m_{i}\right)$ & -3 & 1 & 3 \\
\hline$a_{01}\left(m_{i}\right)$ & -9 & 1 & 3 \\
\hline
\end{tabular}

Na Tabela 4, observa-se que o sistema é estável somente no intervalo $I_{l}$. Então, os valores de $\alpha$ para os quais o sistema é estável pertencem ao intervalo:

$$
-1<\alpha<1 \text {. }
$$

Exemplo 2

Considere o sistema incerto definido por (6), com:

$$
\begin{aligned}
A_{0} & =\left[\begin{array}{cccc}
-1 & 0 & 0 & 0 \\
0 & -2 & 0 & 0 \\
0 & 0 & -3 & 0 \\
0 & 0 & 0 & -4
\end{array}\right], \\
\Delta A & =\left[\begin{array}{cccc}
0 & 1 & 0 & 1 \\
0,5 & 0 & 0,5 & 0 \\
2 & 0 & 1 & 0 \\
0 & -2 & 0 & -1
\end{array}\right] .
\end{aligned}
$$

A matriz do sistema $A=A_{0}+\alpha \Delta A$ é dada por:

$$
A=\left[\begin{array}{cccc}
-1 & \alpha & 0 & \alpha \\
0,5 \alpha & -2 & 0,5 \alpha & 0 \\
2 \alpha & 0 & -3+\alpha & 0 \\
0 & -2 \alpha & 0 & -4-\alpha
\end{array}\right]
$$


e o polinômio característico, calculado através dos Lemas 1 e 2 e da Observação 3, é:

$$
\begin{aligned}
d(s, \alpha)=s^{4} & +10 s^{3}+\left(35-\alpha-1,5 \alpha^{2}\right) s^{2}+\left(50-3 \alpha-6,5 \alpha^{2}\right) s \\
& +\left(24-2 \alpha-8 \alpha^{2}-0,5 \alpha^{3}+0,5 \alpha^{4}\right) .
\end{aligned}
$$

Os valores de $\alpha$ que tornam estável o sistema incerto (6), com as matrizes $A_{o}$ e $\Delta A$ dadas em (14), são determinados pelo Critério de Estabilidade de Routh, como discutido no Exemplo 1. A solução do problema para o Exemplo 2 é:

$$
-2,8996<\alpha<1,6661
$$

\section{Exemplo 3}

Considere o sistema realimentado $\{A-B K C, B, C\}$ definido por (12), com:

$$
A=\left[\begin{array}{cc}
0 & 1 \\
-1 & -2
\end{array}\right], B=\left[\begin{array}{l}
0 \\
1
\end{array}\right], C=\left[\begin{array}{cc}
0,98 & 1
\end{array}\right]
$$

A matriz do sistema de malha fechada $A-B K C$ é dada por:

$$
A-k B C=\left[\begin{array}{cc}
0 & 1 \\
-1-0,98 k & -2-k
\end{array}\right] \mid
$$

e o polinômio característico, calculado através dos Lemas 1 e 2, de acordo com a Subseção 3.1, é:

$$
d(s, k)=s^{2}+(2+k) s+(1+0,98 k) .
$$

Os valores de $k$ que tornam estável o sistema realimentado $\{A-B K C, B, C\}$ definido por (12), com as matrizes $A, B$ e $C$ dadas em (15), são determinados pelo Critério de Estabilidade de Routh, como discutido no Exemplo 1. A solução do problema para o Exemplo 3 é:

$$
k>-1,0204
$$

\section{Conclusões}

Em (TEIXEIRA; ASSUNÇÃO; COVACIC, 2007), foi proposto um método para a determinação da faixa de valores de um parâmetro $k \in \mathbb{R}$ tal que todas as raízes de um polinômio $d(s, k)$ possuam parte real negativa, com base no Critério de Estabilidade de Routh. Neste trabalho, este método foi utilizado para a análise da estabilidade de sistemas incertos $d x(t) / d t=\left(A_{o}+\alpha \Delta A\right) x(t)$ em função do parâmetro $\alpha$. $\mathrm{O}$ método também pode ser aplicado em sistemas realimentados $d x(t) / d t=(A-B K C) x(t)$, com $K=k I$, cuja análise da estabilidade é análoga à análise da estabilidade de sistemas incertos. O método é simples e fornece a solução necessária e suficiente para o problema, pela análise de um conjunto finito de casos, explicitamente definidos no método.

\section{Agradecimentos}

Os autores agradecem o apoio financeiro recebido da FAPESP e do CNPq.

\section{Referências}

BANDYOPADHYAY, B.; ISMAIL, O.; GOREZ, R. Routh Pade approximation for interval systems. IEEE Transactions on Automatic Control, New York, v. 39, n. 12, p. 2454-2456, 1994.

BANDYOPADHYAY, B.; UPADHYE, A.; ISMAIL, O. $\gamma-\delta$ Routh approximation for interval systems. IEEE Transactions on Automatic Control, New York, v. 42, n. 8, p. 1127-1130, 1997.

BARMISH, B. R. Invariance of the strict Hurwitz property for polynomials with perturbed coefficients. IEEE Transactions on Automatic Control, New York, v. 29, n. 10, p. 935-936, 1984.

BIAŁAS, S.; GARLOFF, J. Stability of polynomials under coefficient perturbations. IEEE Transactions on Automatic Control, New York, v. 30, n. 3, p. 310-312, 1985.

BLONDEL, V.; LUNDVALL, C. A rational test for strong stabilization. Automatica, New York, v. 31, n. 8, p. 1197-1198, 1995.

BOSE, N. K. Tests for Hurwitz and Schur properties of convex combination of complex polynomials. IEEE 
Transactions on Circuits and Systems, New York, v. 36, n. 9, p. 1245-1247, 1989.

CHEN, C. T. Analog and digital control system design. Orlando: Saunders College Publishing, 1993.

D’AZZO, J. J.; HOUPIS, C. H. Linear control system analysis and design: conventional and modern. [S. 1.]: McGraw-Hill, 1995.

DORF, R. C.; BISHOP, R. H. Modern control systems. Reading: Addison-Wesley Publishing Company, 1998.

FRANKLIN, G. F.; POWELL, J. D.; EMAMI-NAEINI, A. Feedback control of dynamic systems. Reading: Addison-Wesley Publishing Company, 1994.

GUIVER, J. P.; BOSE, N. K. Strictly Hurwitz property invariance of quartics under coefficient perturbation. IEEE Transactions on Automatic Control, New York, v. 28, n. 1, p. 106-107, 1983.

HWANG, C.; YANG, S. F. Comments on the computation of interval Routh approximants. IEEE Transactions on Automatic Control, New York, v. 44, n. 9, p. 1782-1787, 1999.

HWANG, C.; YANG, S. F. The use of Routh array for testing the Hurwitz property of a segment of polynomials. Automatica, New York, v. 37, n. 2, p. 291-296, 2001.

KUO, B. C. Automatic control system . Upper Saddle River: Prentice-Hall, 1995.
OGATA, K. Engenharia de controle moderno. 4. ed. São Paulo: Prentice-Hall, 2003.

PEÑA, J. M. Characterizations and stable tests for the Routh-Hurwitz conditions and for total positivity. Linear Algebra and its Applications, New York, v. 393, n. 1, p. 319-332, 2003.

ROWLAND, J. Linear control systems: modeling, analysis, and design. New York: Wiley, 1986.

TEIXEIRA, M. C. M.; ASSUNÇÃO, E.; COVACIC, M. R. Proportional controllers: direct method for stability analysis and MATLAB implementation. IEEE Transactions on Education, New York, v. 50, n. 1, p. 7478, 2007.

TEIXEIRA, M. C. M.; MARCHESI, H. F.; ASSUNÇÃO, E. Signal-flow graphs: direct method of reduction and MATLAB implementation. IEEE Transactions on Education, New York, v. 44, n. 2, p. 185-190, 2001.

WEI, K. H.; YEDAVALLI, R. K. Invariance of strict Hurwitz property for uncertain polynomials with dependent coefficients. IEEE Transactions on Automatic Control, New York, v. 32, n. 10, p. 907-909, 1987.

YANG, S. F.; HWANG, C. A test for robust Hurwitz stability of convex combination of complex polynomials. Journal of the Franklin Institute, New York, v. 339, n. 2, p. 129-144, 2002. 